\title{
ESTUDO DA PRODUÇÃO DE PIGMENTOS VERMELHOS POR Monascus ruber CCT 3802 UTILIZANDO CANA DE AÇÚCAR COMO SUBSTRATO EM FERMENTAÇÃO SUBMERSA
}

\author{
D. ISFRAN ${ }^{1}$; J. ALTHOFF ${ }^{2, ; D . E . M O R I T Z ~}{ }^{3}$ E F. VENDRÚSCULO ${ }^{4}$ \\ 1,2,3 Universidade Federal de Santa Catarina, Departamento de Engenharia Química e \\ Engenharia de Alimentos \\ ${ }^{4}$ Universidade Federal de Goiás, Escola de Agronomia, Engenharia de Alimentos. \\ E-mail para contato: deemoritz@gmail.com
}

RESUMO - o objetivo deste trabalho foi avaliar a produção de pigmentos vermelhos por Monascus ruber (CCT 3802) em meio de cultivo submerso contendo caldo de cana diluído (aproximadamente $3^{\circ}$ Brix) e bagaço de cana $\left(10\right.$ g. $\left.\mathrm{L}^{-1}\right)$ desidratado por liofilização como fontes de carbono. Os ensaios foram realizados em frascos Erlenmeyer contendo $250 \mathrm{~mL}$ de volume de meio, a $30{ }^{\circ} \mathrm{C}$ de temperatura, $\mathrm{pH}$ inicial $5,5,150 \mathrm{rpm}$ de frequência de agitação. A fonte de nitrogênio foi a glicina, responsável pela formação de complexos vermelhos hidrossolúveis. Os resultados demonstraram que esta linhagem de fungo produz concentrações elevadas de pigmento vermelho (21,8 U $\mathrm{U}_{\mathrm{Abs} 509)}$, sugerindo que o fungo Monascus utiliza além da glicose como substrato, outros carboidratos oriundos da hidrólise da celulose. Além disso, há poucos relatos bibliográficos sobre a fermentação submersa em caldo-de-cana o que justifica este tipo de estudo.

Palavra chave: biopigmento, Monascus ruber, cana de açúcar.

\section{INTRODUÇÃO}

O estudo e uso de pigmentos naturais nas indústrias de alimentos têm aumentado nos últimos anos devido à toxicidade apresentada pelos de origem artificial. Monascus ruber é um fungo filamentoso conhecido por produzir pigmento vermelho, laranja e amarelo, em diferentes condições de cultivo. Paralelo ao crescimento do mercado de alimentos naturais cresce a preocupação com o aproveitamento de resíduos agroindustriais, constituídos principalmente de carboidratos polimerizados, dos quais a celulose é o mais abundante, compreendendo cerca de dois terços de toda a matéria orgânica existente no planeta (Santos et al., 2012). 
Materiais lignocelulósicos na forma de biomassa de plantas, como o bagaço de cana de açúcar, são os mais abundantes complexos orgânicos de carbono e são constituídos, principalmente, de três componentes: celulose, hemicelulose e lignina (Badhan et al., 2007). Normalmente, diversos tratamentos físico-químicos são realizados para utilização do caldo da cana como meio de cultivo, tais como a filtração, clarificação, precipitação, hidrólise química ou enzimática, etc.; o que muitas vezes, elevam os custos de produção ou aumentam o tempo para obtenção do produto final desejado.

Monascus ruber van Thiehen é um fungo encontrado na natureza que se desenvolve em amido e está presente em muitos alimentos. Esta espécie metaboliza celulose, maltose, frutose e glicose, ocorrendo produção de pigmentos conhecidos como pigmentos Monascus, como mostrado na (Figura 1) (Jung et al., 2003).

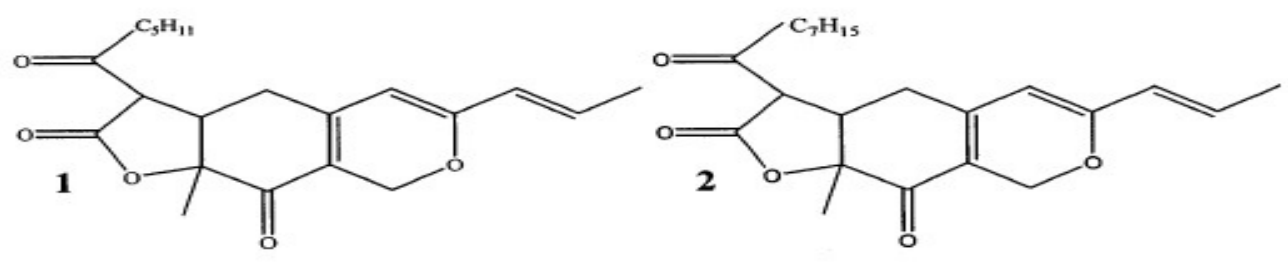

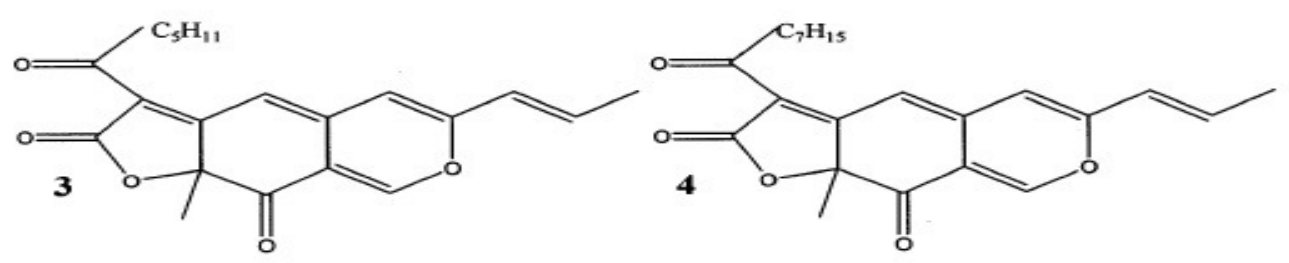

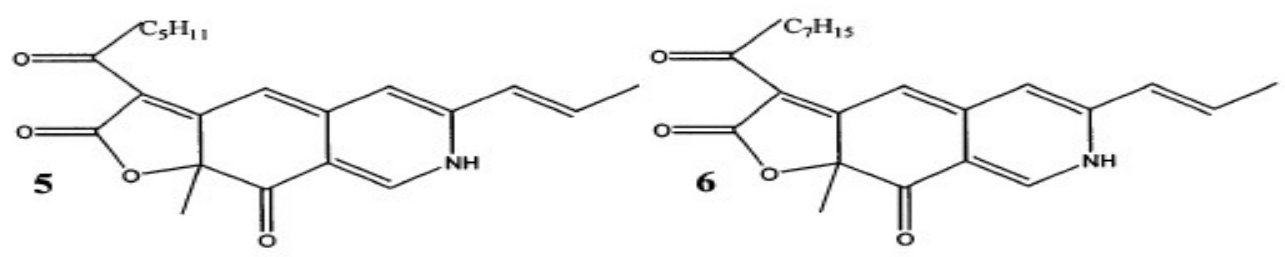

Figura 1 - Estrutura dos seis principais pigmentos produzidos por Monascus: 1, monascin; 2, ankaflavin; 3, monascorubrin; 4, rubropunctatin; 5, monascorubramine; $\mathbf{6}$, rubropunctamine. Fonte: Jung et al. (2003).

Os fungos filamentosos produzem uma ampla diversidade de metabólitos secundários e são explorados em muitos processos industriais, como produção de enzimas, vitaminas, polissacarídeos, pigmentos naturais e lipídeos. Muitas dessas substâncias são produzidas comercialmente e outras apresentam valor potencial para a biotecnologia (Bühler, 2013).

O gênero Monascus é dividido em sete espécies denominadas M. ruber, M. pilosus; $M$. purpureus, M. floridans, M. pallens, M. sanguineus e M. mucoroides. Porém, as espécies de maior importância para a indústria alimentícia são: M. ruber, M. purpureus e M. pilosus (Blanc, 1998). 
Monascus spp utilizam preferencialmente carboidratos como fonte de carbono e energia e produzem compostos como etanol, enzimas e metabólitos secundários pela via policetídica (Hajjaj et al., 1998). Vários metabólitos já foram caracterizados quimicamente tais como pigmentos (Hajjaj et al., 1998; Hu et al., 2012), ácido $\gamma$-aminobutírico (GABA) e ácido dimerúmico (Juzlova, Martinkova; Kren, 1996), a-amilase e glucoamilase (Yoshizaki et al., 2010) e a micotoxina citrinina (Blanc; Loret; Goma, 1995; Jia et al., 2010). Estes metabólitos possuem várias funções, incluindo, antioxidante (Yang et al., 2006), anti-hiperlipidêmica (Choe et al., 2012), anti-inflamatória (Cheng et al., 2012), anticarcinogênica (Akihisa et al., 2005) e com atividade antibacteriana (KIM et al., 2006) e anti-fúngica (Cheng et al., 2011).

Tradicionalmente, os países asiáticos produzem o pigmento em meio sólido através da fermentação do arroz como substrato e poucos trabalhos em cultivo submerso são relatados na bibliografia (Moritz, 2005). Comumente, o pigmento vermelho é o que apresenta maior valor comercial, utilizado como corante alimentar (Yoshizaki et al., 2010). No Brasil, a utilização do pigmento Monascus como aditivo natural ainda não está prevista na legislação (ANVISA, 2014), embora o Red Yeast Rice de origem asiática seja comercializado para todo o mundo.

Baseado neste contexto, o foco deste trabalho foi estudar a produção de biopigmentos vermelhos formados por cultivo submerso a partir do fungo filamentoso Monascus ruber CCT3802 em cana de açúcar como principal fonte de carbono.

\section{MATERIAL E MÉTODOS}

\subsection{Microrganismo e preparo do inóculo}

O microrganismo utilizado nos cultivos foi o fungo filamentoso Monascus ruber CCT3802 (correspondente à cepa ATCC $\mathrm{n}^{\circ}$. 36928), adquirido do Centro de Culturas Tropicais (CCT) da Fundação Tropical de Pesquisas André Tosello (Campinas-SP). O microrganismo foi mantido em meio de caldo de cana, composto por cana de açúcar ( $3^{\circ}$ BRIX), glicina (5,0 g.L -1 $^{-1}$ e ágar bacteriológico (15 g.L -1 $^{-1}$. A manutenção da linhagem foi realizada em tubos de ensaio contendo ágar inclinado a $30^{\circ} \mathrm{C}$, por 7 dias e posteriormente a $4{ }^{0} \mathrm{C}$. O inóculo foi preparado em frascos Erlenmeyer aletados de $1 \mathrm{~L}$ contendo $250 \mathrm{~mL}$ de volume de meio e incubado a $30{ }^{\circ} \mathrm{C}$ por 48 horas em agitador orbital (150 rpm). O pH inicial foi ajustado em 5,5 com ácido fosfórico $85 \%$.

\subsection{Meio de cultivo CBC (Caldo e Bagaço de Cana)}

O meio de cultivo empregado nos experimentos foi composto por cana de açúcar ( $3{ }^{0}$ BRIX), glicina $\left(5,0\right.$ g.L $\left.\mathrm{L}^{-1}\right)$ e bagaço de cana desidratado $\left(1 \mathrm{~g} . \mathrm{L}^{-1}\right)$ preparados em frascos Erlenmeyer aletados de $1 \mathrm{~L}$ contendo $250 \mathrm{~mL}$ de volume de meio e incubado a $30{ }^{\circ} \mathrm{C}$ por 120 horas em agitador orbital $(150 \mathrm{rpm}) . \mathrm{O} \mathrm{pH}$ inicial foi ajustado em 5,5 com ácido fosfórico $85 \%$. 


\subsection{Meio de cultivo AM (Amido de Arroz)}

O meio de cultivo utilizado como controle foi baseado nos estudos de MORITZ (2005) cujos constituintes foram glicose $\left(2\right.$ g.L $\left.\mathrm{L}^{-1}\right)$, glicina $\left(5\right.$ g.L $\left.\mathrm{L}^{-1}\right)$ e amido de arroz $\left(20\right.$ g.L $\left.\mathrm{L}^{-1}\right)$, preparados em frascos Erlenmeyer aletados de $1 \mathrm{~L}$ contendo $250 \mathrm{~mL}$ de volume de meio e incubado a $30{ }^{\circ} \mathrm{C}$ por 120 horas em agitador orbital $(150 \mathrm{rpm}) . \mathrm{O} \mathrm{pH}$ inicial também foi ajustado em 5,5 com ácido fosfórico $85 \%$.

\subsection{Determinação da concentração de carboidratos}

O teste empregado para a determinação de glicose foi o da Biodiagnóstica - Enz color, que utiliza o método da glicose-oxidase e para a determinação dos açúcares redutores (AR) foi utilizado o método de ácido dinitrossalicílico (DNS) segundo (Somogy, 1952) em $540 \mathrm{~nm}$.

\subsection{Determinação de proteínas totais}

O teste empregado para a determinação de proteínas foi o de Bradford (1976) Proteínas Totais. Este método é baseado na interação entre o corante Coomassie Briliant Blue G-250 e macromoléculas de proteínas que contém aminoácidos de cadeias laterais básicas ou aromáticas.

\subsection{Preparo da amostra e determinação de Pigmento}

Após 120 horas de incubação, $200 \mathrm{~mL}$ do fermentado de arroz (AM) e do fermentado de cana de açúcar (CBC) foram liofilizados em liofilizador (LIOBRAS, modelo L101, Brasil) e armazenados em freezer $(\mathrm{BOSH})$ à temperatura de $-24{ }^{\circ} \mathrm{C}$. Para a análise de pigmento em espectrofotômetro foram preparadas soluções aquosas $\left(1\right.$ g.L $\left.\mathrm{L}^{-1}\right)$ e alcoólicas $\left(0,1\right.$ g.L $\left.\mathrm{L}^{-1}\right)$ de cada um dos fermentados e os espectros de absorção dos pigmentos foram analisados por varredura por espectrofotometria UV/Vis (HITASHI U-2900. Japão) utilizando uma cela de $1 \mathrm{~cm}$. A concentração de pigmentos é expressa em Unidades de Absorbância ( $\left.U_{a b s .} \lambda\right)$.

\section{RESULTADOS E DISCUSSÕES}

A Tabela 1 apresenta os resultados dos principais parâmetros cinéticos do processo fermentativo conduzido em caldo e bagaço de cana como principal fonte de carbono e a produção de pigmentos vermelhos em cultivo submerso em frascos agitados (Erlenmeyer), mantidos a $30{ }^{\circ} \mathrm{C}$ por 120 horas em agitador orbital (150 rpm). 
Tabela 1 - Resultados da cinética de Monascus ruber CCT 3802 utilizando cana de açúcar como substrato em fermentação submersa.

\begin{tabular}{|c|c|c|c|c|}
\hline \multirow{2}{*}{$\begin{array}{l}\text { Tempo } \\
\text { (h) }\end{array}$} & \multicolumn{2}{|c|}{ Carboidratos } & \multirow{2}{*}{$\begin{array}{l}\text { Proteínas Totais } \\
\text { mg.L }\end{array}$} & \multirow{2}{*}{$\mathrm{pH}$} \\
\hline & $\begin{array}{l}\text { Conc. AR } \\
\text { g.L }\end{array}$ & Conc. Gli g.L & & \\
\hline 0 & 52,02 & 31,54 & 23,18 & 5,5 \\
\hline 24 & 41,40 & 35,84 & 118,34 & 5,6 \\
\hline 48 & 32,72 & 29,83 & 61,38 & 7,1 \\
\hline 72 & 28,43 & 25,89 & 54,78 & 7,2 \\
\hline 96 & 17,25 & 13,03 & 83,36 & 7,3 \\
\hline 120 & 9,53 & 7,05 & 37,87 & 6,9 \\
\hline
\end{tabular}

A biomassa final foi de 11,34 g. $\mathrm{L}^{-1}$ e o $\mathrm{Y}_{\mathrm{X} / \mathrm{S}}$ - coeficiente de rendimento em biomassa (g biomassa/g de açúcar consumido) foi de $0,22 \mathrm{~g} \cdot \mathrm{g}^{-1}$. Resultados similares quanto a biomassa e coeficiente de rendimento em biomassa foram relatados por Moritz (2005) utilizando amido de arroz e Pisareva e Kujumdzieva (2010) utilizando glicose como principal fonte de carbono.

$\mathrm{O} \mathrm{pH}$ do caldo fermentativo gerado pelo fungo Monascus ruber no meio $\mathrm{CBC}$ variou entre 5,5 (inicial) e 6,9 (final). Para o meio de cultivo utilizando amido de arroz (AM) como substrato e mesmo $\mathrm{pH}$ inicial foi encontrado um $\mathrm{pH}$ final de 8,2. Esta diferença de valores finais de $\mathrm{pH}$ sugerem que mais metabólicos ácidos são formados pela degradação do bagaço e da cana de açúcar, diferentemente da degradação do amido do arroz.

Os pigmentos oriundos do processo fermentativo foram qualificados por espectroscopia de varredura entre os comprimentos de onda: $200 \mathrm{~nm}$ e $700 \mathrm{~nm}$. O resultado da análise espectral (Figura 2) do pigmento dissolvido em água na concentração de 1 g.L.-1 e em etanol na concentração de 0,1 g.L.- obedeceram a Lei de Beer, como expresso pela Equação 1.

$$
A=\varepsilon b c(1)
$$

Onde $A=$ absorbância; $\varepsilon=$ coeficiente de absortividade molar do meio (L.mol ${ }^{-1} \cdot \mathrm{cm}^{-1}$ ); $b=$ espessura do volume de observação $(1 \mathrm{~cm})$ e $c=$ concentração de átomos no estado fundamental. 


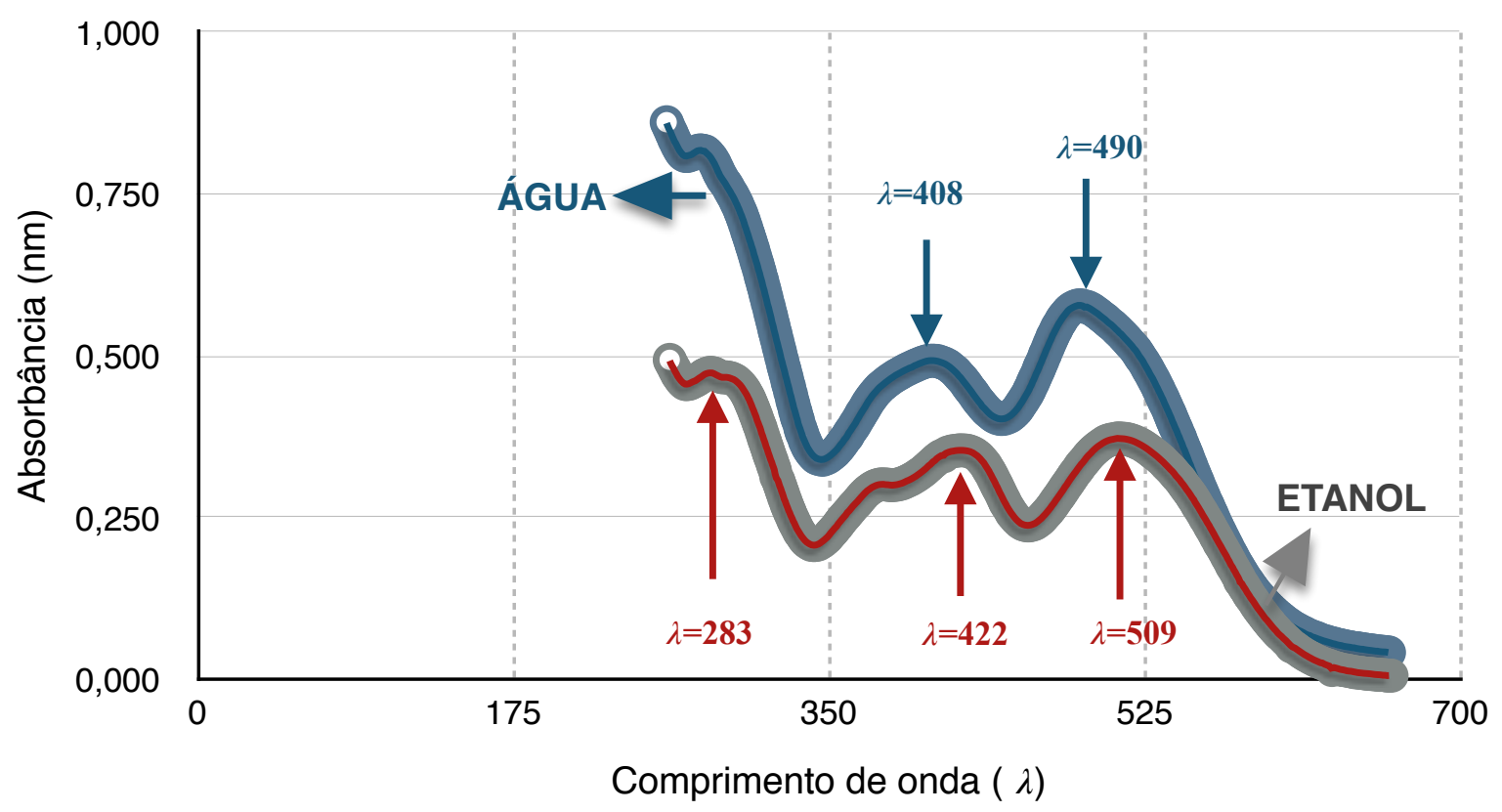

Figura 2 - Espectro de varredura em espectrofotômetro entre os comprimentos de onda: $200 \mathrm{~nm}$ - $700 \mathrm{~nm}$ de pigmento Monascus dissolvido em água na concentração de 1 g.L.-1 e dissolvido etanol na concentração de 0,1 g. $\mathrm{L}^{-1}$. O fator de diluição (FD) utilizado foi FD 3 para água e FD 4 para etanol. Os comprimentos de onda máximos ( $\chi$ max) estão apresentadas no gráfico ( $\lambda=283 \mathrm{~nm} ; . \lambda=422 \mathrm{~nm}$ e $\lambda=509 \mathrm{~nm}$ para etanol (em vermelho) e $\lambda=408 \mathrm{~nm}$ e $\lambda=490 \mathrm{~nm}$ (em azul) para água como solvente.

É possível observar através deste gráfico espectral que há distinção entre as faixas de cor absorvidas quando se modifica o solvente utilizado. Quando o pigmento Monascus foi dissolvido em etanol verifica-se duas faixas espectrais na região Visível (Vis) $\lambda=422 \mathrm{~nm}$ e $\lambda=509 \mathrm{~nm}$ e, que correspondem à transmissão da cor amarelo e púrpura, respectivamente. A outra na faixa espectral corresponde a região Ultra-Violeta $U V(\lambda=283 \mathrm{~nm})$. Já para a água como solvente, apenas duas outras faixas Vis foram observadas $\lambda=408 \mathrm{~nm}$ e $\lambda=490 \mathrm{~nm}$, que, segundo Skoog et al. (2007) correspondem respectivamente, à faixa de cor transmitida verdeamarelo $(\lambda=400-435 \mathrm{~nm})$ e vermelho $(\lambda=490-500 \mathrm{~nm})$. Apesar de solventes distintos, a faixa de cor transmitida como verde-amarelo foram detectadas tanto na água quanto no etanol.

Segundo Skoog et al. (2007) as absortividades são funções dependentes de variáveis como o tipo de solvente, a composição da solução e da temperatura. Por causa da variação da absortividade com esses parâmetros, nunca é muito prudente tornar-se dependente de valores tabelados na literatura para realizar uma análise quantitativa. Portanto, uma solução padrão do analito no mesmo solvente e à mesma temperatura é empregada para se obter a absortividade no momento da análise. Em se tratando do Pigmento Monascus, cujo padrão é inexistente 
comercialmente, por apresentar mais de um cromóforo e ainda, os espectros de absorção desses cromóforos se sobreporem numa dada extensão de comprimento de onda, não é possível afirmar que a região de absorbância máxima $(\lambda) 410 \mathrm{~nm}$ corresponde ao pigmento Monascus amarelo, que o comprimento de onda $(\lambda) 420 \mathrm{~nm}$ corresponde ao pigmento laranja ou o comprimento de onda $(\lambda) 510$ corresponda ao pigmento vermelho como afirmado por alguns autores (Juzlova, Martinkova; Kren, 1996, Hajjaj et al., 1998).

Os pigmentos oriundos do processo fermentativo foram quantificados baseado na avaliação espectral descrita anteriormente. As concentrações máximas encontradas nos comprimentos de onda $(\lambda) 509 \mathrm{~nm}$ e $(\lambda) 420 \mathrm{~nm}$ foi de 21,77 U $\mathrm{U}_{\mathrm{Abs} 509}$. e 7,21 U Ubs420 (pigmento púrpura e amarelo, respectivamente, dissolvidos em etanol). Já para os pigmentos dissolvidos em água, as concentrações máximas encontradas nos comprimentos de onda ( $\lambda$ ) $490 \mathrm{~nm}$ e $408 \mathrm{~nm}$ foram de 15,40 $\mathrm{U}_{\mathrm{Abs} 490}$. e 5,56 $\mathrm{U}_{\mathrm{Abs} 408}$ (pigmento vermelho e pigmento amarelo, respectivamente).

Segundo Vendruscolo (2009) os pigmentos gerados pelo fungo Monascus ruber apresentam comportamentos diferentes, principalmente em função do tipo de substrato utilizado. O pigmento vermelho é solúvel em água e, conseqüentemente, solúvel no meio de cultivo, composto por níveis superiores a $95 \%$ de água. Devido à afinidade por grupos amino, os pigmentos de Monascus estão associados freqüentemente a proteínas ou à parede celular, formando um complexo que pode ser de difícil extração na fase aquosa e facilitada na fase alcoólica, o que pode justificar a diferença espectral observada na Figura 2.

\section{CONCLUSÃO}

Os resultados encontrados neste trabalho são importantes, tanto para o emprego do cultivo submerso, como para fornecer dados bastante úteis para novos estudos utilizando cana de açúcar para a produção de pigmentos vermelhos.

A concentração elevada de proteínas totais aliada ao consumo de substrato indica uma hipótese de produção de enzimas importantes, principalmente as celulolíticas que possuem grande interesse comercial na atualidade e que merecem ser mais bem investigados.

\section{REFERÊNCIAS}

BADHAN, A. K.; Chadha, B. S.; Kaur, J.; Saini, H. S.; Bhat, M. K. (2007). Production of multiple xylanolytic and cellulolytic enzymes by thermophilic fungus Myceliophthora sp. IMI 387099.;Bioresour. Technol.,98p, 504-510. 2007.

BLANC, P. J.. Les Pigments Rouges de Monascus. Biofutur, v. 184, p. 13-17. 1998.

BLANC, P. J.; LORET, M. O.; GOMA, G. Production of citrinin by various species of Monascus. Biotech. Lett., v. vol. 17, n. 3, p. 291-294. 1995 
BÜHLER, R. M. M. Produção de Pigmentos por Monascus ruber utilizando Co-produtos da produção de Biodiesel. 182 (Doutorado). Programa de Pós-graduação em Engenharia de Alimentos, UFSC, Florianópolis, SC. 2013.

CHENG, M.-J. et al. Chemical constituents from the fungus Monascus purpureus and their antifungal activity. Phytoch. Lett., v. 4, n. 3, p. 372-376. 2011.

CHENG, M.-J. et al. Antiinflammatory compounds from Monascus pilosus-fermented rice. Phytoch. Letters, v. 5, n. 1, p. 63-67. 2012.

CHOE, D. et al. Evaluation of the amine derivaties of Monascus pigment with anti-obesity activities. F. Chemistry, v. 134, n. 1, p. 315-323. 2012.

HAJJAJ, H. et al. Sampling techniques and comparative extraction procedures for quantitative determination of intra- and extracellular metabolites in filamentous fungi. Fems Microb. Letters, v. 164, n. 1, p. 195-200. 1998.

HU, Z. et al. Export of intracellular Monascus pigments by two-stage microbial fermentation in nonionic surfactant micelle aqueous. J. of Biotechnol. n. 162, p. 202-209. 2012.

JIA, X. et al. Elimination of the mycotoxin citrinin production in the industrial important strain Monascus purpureus SM001. Metabolic Eng., v. 12, n. 1, p. 1-7. 2010.

JUNG, K,; CHULYOUNM, K.; K IM,K.; SHIN, C.S. Color Characteristics of Monascus Pigments Derived by Fermentation with Various Amino Acids. J. of Agricultural and Food Chem.. V. 51; p. 1302-1306. 2003.

JUZLOVA, P. L.; MARTINKOVA, L.; KREN, V. (1996). Secundary metabolites of the fungus Monascus. J. Industrial Microbiol., v. 16, p. 163-170.

KIHISA, T. et al. (2005). Azaphilones, furanoisophthalides, and amino acids from the extracts of Monascus pilosus - fermented rice (red-mold rice) and their chemopreventive effects. J. of Agricultural and Food Chem., v. 53, p. 562-565.

KIM, C. et al. (2006). Antimicrobial activities of amino acid derivaties of monascus pigments. FEMS Microbiol. Lett. App. Microbiol., v. 264, p. 117-124.

MORITZ, D. E. Produção de pigmento monascus por Monascus ruber CCT 3802 em cultivo submerso. Curso de Engenharia Química, Departamento de Engenharia Química e Engenharia de Alimentos. Florianópolis: Universidade Federal de Santa Catarina. Doutorado: 134 p. 2005.

PISERAVA, E.I.; KUJUMDZIEVA, A.V. Influence Of Carbon And Nitrogen Sources On Growth And Pigment Production By Monascus pilosus Strain. Biotechnol. \& Biotechnol. p. 501-506. 2010. 
SANTOS, F.A., QUEIRÓZ, J.H., COLODETTE, J.L., FERNANDES, S.A.; GUIMARÃES, V.M.; REZENDE, S.T. Potencial da Palha de Cana-de-açúcar Para Produção de Etanol. Quím. Nova. vol. 35, n5, p. 1004-1010. 2012.

SKOOG, D.A; et al. Fundamentos da Química Analítica, Tradução da $8^{\mathrm{a}}$ edição norte americana. São Paulo, Ed.Thomson, 2007.

VENDRÚSCULO, F. Produção de Pigmento Vermelho a partir do Pigmento Laranja Produzido por Monascus ruber CCT 3802. Curso de Engenharia Química, Departamento de Engenharia Química e Engenharia de Alimentos. Florianópolis: UFSC. Doutorado: 241 p. 2009.

WANG, S. L.; HSIAO, W. J.; CHANG, W. T. Purification and characterization of an antimicrobial chitinase extracellularly produced by Monascus purpureus CCRC31499 in a shrimp and crab shell powder medium. J. of Agr. and Food Chemistry, v. 50, n. 8, p. 2249-2255, Apr 102002.

YANG, J.-H. et al. Antioxidant properties of methanolic extracts from monascal rice. Food Science and Technology, v. 39, n. 7, p. 740-747,, 2006.

YOSHIZAKI, Y. et al. Characterization of glucoamylase and a-amilase from Monascus anka: Enhanced production of a-amylase in red koji. J. of Bioscience and Bioeng.. V. 110, n. 6, p. 670-674, 2010. 